Ann. Génét. Sél. anim., I969, 1 (3), 227-235.

\title{
DONNÉES COMPLÉMENTAIRES SUR DES ANOMALIES DE SÉGRÉGATION AU LOCUS $R$ CHEZ LA POULE, LORSQUE LE PĖRE EST HÉTÉROZYGOTE
}

\author{
P. MÉRAT \\ Station centrale de Génétique animale, \\ Centre national de Recherches zootechniques, 78-Jouy-en-Josas \\ Institut national de la Recherche agronomique
}

SOMMAIRE

Des résultats supplémentaires sont présentés à propos d'une anomalie du rapport de ségrégation au locus $R$ (crête en rose /crête simple) chez la poule. Ils renforcent l'hypothèse de a fertilisation sélective "émise précédemment. De plus, les résultats de la ségrégation conjointe aux loci $R$ et $C$ suggèrent la possibilité d'une "interaction " ou " compétition directe " entre spermatozoïdes $R C$ et $r C$ en présence d'un ovule $\mathrm{X}$.

\section{INTRODUCTION}

Dans une série d'articles (MÉRAT, I962, I963a, I966a), nous avons fait mention de proportions mendéliennes anormales obtenues dans des accouplements pedigree du type đơ $R r$ (crête en rose) $\times$ \%̊ $r r$ (crête simple). Il s'agissait d'un défaut de descendants mâles à crête en rose et d'un excès significatif de mâles à crête simple par rapport à la proportion théorique $\mathrm{I} / \mathrm{I}$.

L'anomalie en cause ne doit pas être postérieure à la formation des zygotes. En effet, le taux d'éclosion dans les familles présentant la proportion la plus perturbée est élevé dans l'ensemble et n'est pas inférieur à celui des autres familles. En outre, il existe non seulement un défaut de mâles à crête en rose, mais aussi un excès de mâles à crête simple. D'autre part, une dominance variable de l'allèle $R$ paraît exclue : par test de descendance, jamais un seul coq à crête simple issu de familles à ségrégation perturbée ne s'est révélé être du génotype $R r$ (MÉRAT, I962). 


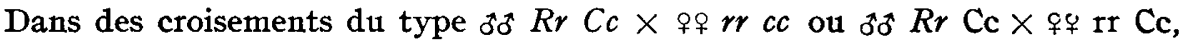
présentant en même temps une ségrégation pour $\mathrm{C} / \mathrm{c}$ (présence/absence de pigmentation du plumage), l'excès de crêtes simples et le défaut de crêtes en rose chez les mâles étaient restreints aux oiseaux colorés (Cc ou $\mathrm{CC}$ ) : la proportion des types de crête était normale parmi les descendants $\mathrm{cc}$. Ce résultat renforçait l'hypothèse précédente de ségrégations anormales antérieures à la formation du zygote (MÉRAT, Ig66b).

Ultérieurement, nous avons découvert un autre article faisant état du rapport de ségrégation de crêtes simples ou en rose détaillé par sexe (WARREN, I948). Il s'agissait de croisements où l'un des parents était hétérozygote, l'autre homozygote récessif, mais l'auteur signalait que, dans la majorité des cas, l'hétérozygote était le mâle.

Ces données, recueillies en vue d'une étude de linkage, n'étaient pas analysées sous l'angle qui nous intéresse ici. Elles comprenaient au total I I86 mâles à crête en rose contre I 3 I 4 à crête simple; les nombres correspondants chez les femelles étaient I 244 et I 2 I5.

Les proportions sont assez voisines des nôtres. Comme dans nos résultats, on peut constater que le rapport de ségrégation des types de crête ne s'écarte significativement de I / I ni sur les deux sexes groupés, ni chez les femelles, alors que les mâles présentent un excès de crêtes simples $\left(\chi^{2}=6,554, \mathrm{P} \simeq 0,0 \mathrm{I}\right)$. La différence de proportion suivant le sexe est significative au seuil I p. Ioo ( $\chi^{2}$ "interaction " $=6,557)$ et $1^{\prime}$ on note un excès de mâles parmi les crêtes simples, $\left(\chi^{2}=3,876\right.$, $\mathrm{P} \simeq 0,05)$ et un léger défaut du même sexe parmi les crêtes en rose $\left(\chi^{2}=1,384\right.$, N.S.).

Il est donc possible que le phénomène mis en évidence dans notre population ne soit pas un cas isolé.

Des données plus récentes nous permettent de confirmer et de préciser davantage nos premières hypothèses.

\section{MATÉRIEL ET MÉTHODES}

Des précisions sur les cheptels étudiés, leur origine (croisement initial entre Rhode-Island, Wyandotte et Gátinaise) et leurs effectifs par année (de 1954 à 1966) ont été données dans nos articles antérieurs (MÉrat, 1962, 1966a). Le type de crête est diagnostiqué sans ambiguité à la naissance, et, depuis 1959 , sur les embryons après 18 jours d'incubation. Quant au sexe, il est déterminé à l'âge de 8 semaines et vérifié 2 semaines plus tard sur les animaux vivants. Pour les poussins et les embryons morts dont le stade de développement le permet, il est précisé par autopsie depuis 1959 inclusivement.

\section{RESULTATS}

\section{Données supplémentaires sur les proportions globales (population "principale 》)}

En totalisant nos résultats jusqu'en I968, on aboutit aux nombres suivants

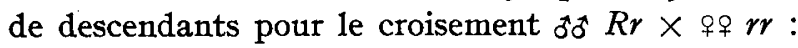

- chez les mâles, 2988 à crête en rose, 3259 à crête simple;

- chez les femelles, 3037 à crête en rose, 298 I à crête simple.

La même tendance générale persiste et les mêmes conclusions sont suggérées. 


\section{Résultats sur une souche Wyandotte blanche non fixée homozygote pour $R$}

En plus de la population étudiée ci-dessus, nous avons obtenu des données sur une souche de Wyandottes non fixée pour l'allèle $R$ (Élevage SARGas et Domaine du Magneraud). Les nombres de crêtes simples et en rose dans la descendance de croisements $\delta \sigma^{*} R r \times$ 우 $r r$ y sont les suivants :

Mâles : 260 à crête en rose, 2 I9 à crête simple.

Femelles : I92 à crête en rose, 252 à crête simple.

La proportion diffère encore suivant le sexe $\left(\chi^{2}\right.$ de contingence égal à II,244, $\mathrm{P}<0,00 \mathrm{I})$; mais il $\mathrm{y} \mathrm{a}$, contrairement à notre première population, un excès de crêtes en rose chez les mâles et un défaut chez les femelles, avec, dans l'ensemble, une proportion des types de crête voisine de l'égalité. En outre, la proportion des sexes est différente de I/I à l'intérieur de chaque type de crête (tabl. I).

\section{TABLEAU I}

Proportion des sexes par types de crête dans une souche Wyandotte

\begin{tabular}{|c|c|c|c|c|c|}
\hline $\begin{array}{l}\text { Types } \\
\text { de crête }\end{array}$ & $\begin{array}{l}\text { Non } \\
\text { ठే }\end{array}$ & de & $\%$ des & de la porportion $I / I$ & Probabilité \\
\hline $\begin{array}{l}\text { Simple } \\
\text { en rose }\end{array}$ & $\begin{array}{l}219 \\
260\end{array}$ & $\begin{array}{l}252 \\
192\end{array}$ & $\begin{array}{l}46,5 \\
57,5\end{array}$ & $\begin{array}{r}2,313 \\
10,230\end{array}$ & $\begin{array}{l}\text { N.S. } \\
<\text { o,oOI }\end{array}$ \\
\hline
\end{tabular}

Il semble, ici aussi, difficile d'expliquer les faits par un simple manque de certaines catégories zygotiques : l'excès de femelles chez les crêtes simples ne se retrouve pas dans des croisements où les deux parents sont à crête simple, et l'excès de mâles constaté chez les crêtes en rose ne s'observe pas dans les croisements donnant des descendants tous à crête en rose.

Dans chaque sexe, les proportions diffèrent d'une façon hautement significative $(\mathrm{P}<\mathrm{O}, \mathrm{OI})$ de celles de notre première population. La raison de cette différence n'est pas connue. On peut seulement remarquer que, chez la Wyandotte, tous les animaux étaient homozygotes pour le blanc récessif $(c c)$, que nous avons déjà trouvé interférer avec la ségrégation des types de crête.

\section{Fluctuations du rapport de ségrégation dans le temps, pour la population principale}

Dans les familles à rapport de ségrégation anormal chez les mâles, ce rapport semble varier au cours du temps. Dans la population principalement étudiée, les familles présentant une proportion significativement différente de I/I au seuil 5 p. roo chez les mâles, correspondaient à 9 pères, répartis sur 6 années. Pour chacun, nous avons calculé le $\chi^{2}$ d'hétérogénéité de cette proportion entre semaines succes- 
sives d'éclosion. La somme de ces $\chi^{2}$ " entre semaines intra-pères " est égale à 82,425 pour 53 degrés de liberté, ce qui conduit à une valeur $t=\sqrt{2 \chi^{2}}-\sqrt{2 v-I}$ égale à 2,59 (probabilité voisine de 0,005 ). En corrigeant le $\chi^{2}$ pour chaque père, compte tenu de ce que la proportion totale pour ce dernier diffère sensiblement de $I / I$, pour avoir une approximation plus exacte $d u \chi^{2}$ de contingence, on obtient encore une valeur de $t$ égale à 2,33 ( $\left.\mathrm{P} \simeq 0,0 \mathrm{I}_{5}\right)$.

La réalité de ces fluctuations dans le temps est donc vraisemblable, sans que l'on puisse pour l'instant les interpréter ( ${ }^{\mathbf{1}}$ ).

\section{Observations complémentaires sur la double ségrégation aux loci $R$ et $C$ dans la population principale.}

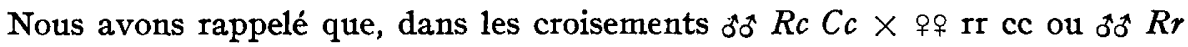
$C c \times q q r r C c$, seule la proportion des crêtes chez les mâles colorés $(C c$ ou $C C)$ s'écarte de I / I, et ce de façon hautement significative (MÉRAT, I966b, tabl. I et 2) ( ${ }^{2}$ )

Autrement dit, il existe un excès de mâles à crête simple et de femelles à crête en rose, limité aux animaux colorés, comme l'indique le tableau 2.

TABLEAU 2

"Sex ratio " comparée suivant le type de crête et de plumage, sur le total des données

\begin{tabular}{|c|c|c|c|c|}
\hline Phénotype & $\begin{array}{c}\text { Nombre } \\
\text { de } \delta \delta^{\top}\end{array}$ & $\begin{array}{c}\text { Nombre } \\
\text { de } \$ q\end{array}$ & $\begin{array}{c}\text { Pourcentage } \\
\text { des } \delta^{\top} \delta^{*}\end{array}$ & $\begin{array}{c}\chi^{2} \text { vis-à-vis } \\
\text { de la proportion } \\
\text { I / I pour les sexes }\end{array}$ \\
\hline $\begin{array}{l}(\mathrm{CR}) \\
(\mathrm{Cr}) \\
(\mathrm{cR}) \\
(\mathrm{cr})\end{array}$ & $\begin{array}{l}325 \\
435 \\
282 \\
266\end{array}$ & $\begin{array}{l}363 \\
348 \\
260 \\
260\end{array}$ & $\begin{array}{l}47,2 \\
55,6 \\
52,0 \\
50,6\end{array}$ & $\begin{array}{l}2,100 \\
9,667 \\
0,893 \\
0,070\end{array}$ \\
\hline
\end{tabular}

Cette observation, qui fait ressortir encore plus l'excès d'une catégorie (mâles colorés à crête simple), confirme qu'il ne doit pas s'agir d'une mortalité zygotique plus élevée dans certaines classes $\left({ }^{3}\right)$.

De ce point de vue, une vérification complémentaire est possible. Nos proportions indiquent un excès de mâles $(C r)$ non seulement par rapport aux $(C R)$, mais aussi par comparaison avec les (cr) (tabl. 3).

(1) Des fluctuations du rapport de ségrégation dans le temps sont également suggérées pour le croisement $\delta^{\star} \widehat{R r} \times$ 우 $R r$. En nous limitant, pour ce croisement, aux familles de même père présentant une interaction significative au seuil $5 \mathrm{p}$. Ioo entre sexe et type de crête pour les proportions, évaluant le $\chi^{2}$ d'hétérogénéité entre semaines d'éclosion dans chaque famille, et additionnant ces $\chi^{2}$, nous obtenons, sur les sexes groupés et sur les mâles seuls, des valeurs non significatives; sur les femelles, cependant, le $\chi^{2}$ obtenu ainsi est de 41,8 pour 26 degrés de liberté $(P \simeq 0,02)$.

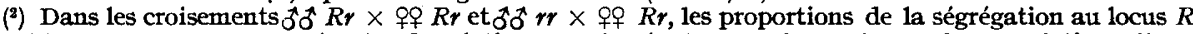
ne semblent pas, par contre, présenter de relation avec le génotype au locus $C$, sur des populations, il est vrai, différentes de celle étudiée ici.

$\left({ }^{3}\right)$ Si l'on se limite aux familles ayant présenté un excès global de crêtes simples, significatif au seuil 5 p. roo, parmi les mâles colorés, on observe une proportion de 59,7 p. roo de mâles parmi les zygotes colorés à crête simple. 
TABLEAU 3

Proportion des mâles colovés et blancs à crête simple dans les croisements étudiés

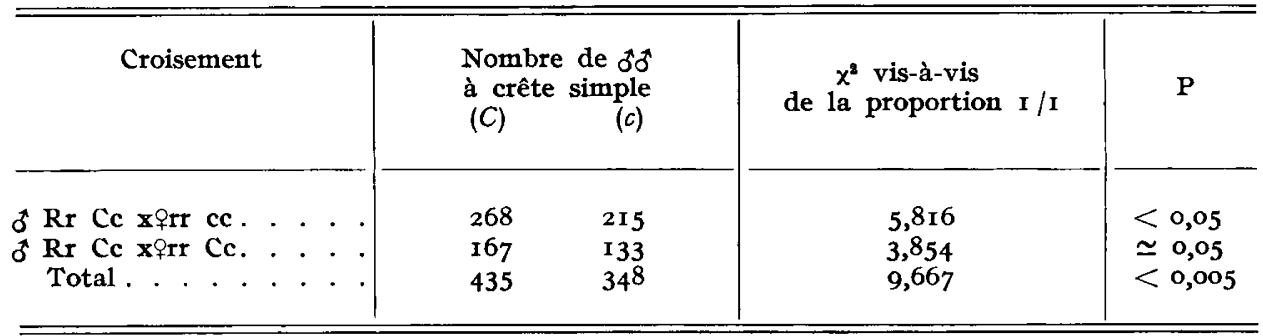

Une différence de mortalité zygotique devrait, en moyenne, se retrouver

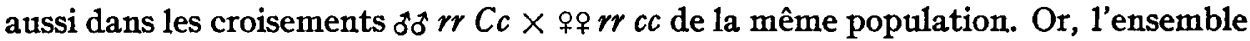

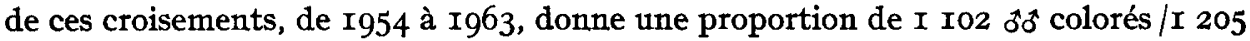
đơ blancs, c'est-à-dire en fait un léger excès des seconds $\left(\chi^{2}=4,599, \mathrm{P}<0,05\right)$, et pas d'hétérogénéité significative entre familles de pères $\left(\chi^{2}=43,226\right.$ pour 52 degrés de liberté), ce qui ne s'accorde pas avec l'hypothèse envisagée. Il en est de même du croisement $C c \times C c$ : quand les 2 parents sont à crête simple, la proportion des descendants mâles colorés aux blancs est de $84 \mathrm{I} / 330$, ne reflétant donc en aucune façon un excès de colorés, mais au contraire un excès de blancs ( $\chi^{2}$ $=6,320, \mathrm{P}<0,05)$.

Une conclusion analogue n'a pas pu être tirée dans le cas où le père est homozygote $R R$ pour la crête en rose, les croisements correspondants ayant été peu nombreux.

\section{DISCUSSION}

\section{Fertilisation sélective.}

Les observations précédentes indiquent comme fait saillant, sur la population principale étudiée, une ségrégation anormale limitée à certaines catégories de mâles. $L_{L}$ conclusion est analogue pour la deuxième population envisagée, quoique le sens des phénomènes ne soit pas le même.

Outre les hypothèses de mortalité zygotique et de pénétrance incomplète, nous avons éliminé celle d'anomalies de la répartition du chromosome $X$ dans l'ovule ou le globule polaire, d'anomalies dans la détermination du sexe, ou de pseudogamie (MÉRAT, I963b).

On ne peut envisager non plus la production de spermatozoïdes anormaux " $O R$ " (O désignant la perte d'un chromosome $\mathrm{X}$ ) qui, avec des ovules $\mathrm{X} r$, donneraient des filles $\mathrm{R} r$, les spermatozoïdes $\mathrm{X} r$ se trouvant en excès, d'où production de mâles à crête simple en proportion supérieure aux prévisions. Cela supposerait un excès global de femelles, avec, parmi elles, excès de crêtes en rose et une létalité ou infertilité par formation de zygotes privés de chromosome $\mathrm{X}$, toutes choses qui n'apparaissent pas dans nos résultats. 
Il faut écarter, de même, la possibilité de formation de spermatozoïdes $\mathrm{XXr}$ (par non disjonction de l'X et ségrégation préférentielle avec le chromosome porteur de r), donnant des zygotes XXrr avec des ovules $\mathrm{O} r$; ceci impliquerait un excès global de mâles et une létalité ou des anomalies correspondant aux zygotes XXX formés $\left.{ }^{1}\right)$.

Nous sommes donc confirmés dans l'interprétation déjà proposée (MÉrAT, I963b) d'une " pénétration sélective " du spermatozoïde dans l'ovule du type "par facilité ", selon la terminologie de BATEMAN (1960), cette explication étant renforcée par les résultats de la ségrégation simultanée avec $C / c$.

\section{Compétition " directe " entre spermatozoides}

Cette ségrégation apporte un élément nouveau, que nous n'avons pas encore mis en lumière : c'est la possibilité d'une "interaction" directe entre spermatozoïdes de génotypes différents, ou plutôt, compte tenu de l'ovule, d'une interaction du deuxième ordre ovule - spermatozoïde - spermatozoïde.

En effet, et en nous limitant au cas où un ovule porteur du chromosome $\mathbf{X}$ est présent, il n'est pas très satisfaisant de supposer une simple différence de classement " en valeur absolue " entre les 4 types de spermatozoïdes $C R, C r, c R$ et $c r$, car les catégories $c R$ et $c r$ se placeraient à égalité, et à peu près à mi-chemin entre les deux autres, ce qui constituerait une coïncidence assez particulière.

Les proportions de mâles $(C)$ par rapport aux $(c)$ dans des croisements ${ }^{\star} \delta$ rr $\times$ 우 rr nous fournissent d'ailleurs un terme de comparaison. Dans nos familles à ségrégation significativement anormale pour $R$ et $r$, et présentant en même temps une disjonction pour $C$ et $c$, les mâles $(C r)$ sont en excès non seulement par rapport aux $(C R)$, mais aussi, secondairement, vis-à-vis des $(c r)$. Cet excès est hautement significatif $(\mathrm{P}<0,0 \mathrm{I})$. S'il s'agit d'une différence " intrinsèque " de "valeur sélective ", elle devrait se traduire de la même façon — au moins dans certaines familles — dans les croisements $\sigma^{*} \sigma^{*} C c r r \times q q c c r r$ (ou ơ $C c r r \times q q C c r r$ ) issus de la même population. Or, sur des nombres relativement grands, les proportions dans ces deux croisements, comme mentionné plus haut, n'indiquent aucun excès de mâles colorés et aucune hétérogénéité entre familles $\left({ }^{2}\right)$.

La " compétition " semblerait donc limitée à celle des gamètes mâles $C R$ vis$\grave{a}$-vis des $C r$, donc à une partie seulement des spermatozoïdes, comme si la "valeur sélective "d'un spermatozoïde, vis-à-vis de l'ovule $\mathrm{X}$, dépendait de la " présence " ou du " voisinage " de spermatozoïdes d'un autre génotype spécifié $\left({ }^{3}\right)$.

On ne pourrait écarter cette hypothèse qu'en admettant que les "valeurs sélectives intrinsèques " des gamètes mâles sont modifiées en sens contraire l'une de l'autre, si le génotype du mâle est $\operatorname{Rr} C c$ au lieu de $r r C c$.

(1) Les considérations qui précèdent sont encore confirmées par les proportions absolument normales observées pour le gène lié au sexe $\mathrm{S}$, $\mathrm{s}$, qui se trouvait en ségrégation dans certaines des familles étudiées pour le locus $R$.

( ${ }^{2}$ Un raisonnement analogue devrait pouvoir être fait à partir de la comparaison de la proportion des zygotes $(C R)$ et $(C R)$ dans nos familles à ségrégation anormale pour la crête, et dans les croisements Cc $R R \times$ cc RR, mais nous n'avons pu réunir jusqu'à présent que peu de données relatives à ces derniers.

$\left({ }^{3}\right)$ En d'autres termes, la proportion $\mathrm{I} / \mathbf{I}$ entre zygotes (C) et (c) d'une part, et celle différente de $\mathrm{I} / \mathrm{I}$ entre $(C R)$ et $(C r)$, font penser au schéma d'un tirage aléatoire entre gamètes $C$ et $c$, avec des chances égales, combiné à un tirage entre $\mathrm{CR}$ et $\mathrm{Cr}$ parmi les $\mathrm{C}$, avec des probabilités, cette fois, inégales. 
Selon l'hypothèse suggérée, les phénomènes pour un type de gamètes apparaissent, en fin de compte, différents suivant les autres types avec lesquels il est en " compétition ". Une telle possibilité n'a jamais, à notre connaissance, été même envisagée. Voyons, d'abord, si elle est concevable.

L'ovule est impliqué dans l'interaction postulée, qui devrait donc se situer soit dans son voisinage proche, soit à son intérieur, la polyspermie existant chez les oiseaux (PIko, I96I). Bien des modalités seraient a priori imaginables : chemotactisme (ARNOLD, I958), réactions de nature immunologique (TYLER, I96I)... Mais la notion supplémentaire de " réaction directe " entre spermatozoïdes devrait impliquer, semble-t-il, un voisinage spécialement étroit, et le fait qu'elle soit limitée à 2 types sur 4 s'expliquerait au mieux, à première vue, si les choses se passent à l'intérieur de l'ovule. On conçoit, dans ce cas, que la " compétition " soit restreinte précisément aux noyaux spermatiques ayant pénétré le gamète femelle.

Il est encore difficile de proposer une vérification physiologique de cette hypothèse de travail, compte tenu de ce que les conditions favorisant 1'apparition du phénomène en cause ne sont pas connues encore avec précision et que, par là, il n'est pas reproductible à volonté.

Précisément, si la polyspermie en est le support, elle devra en être aussi la condition. Si elle est inexistante, c'est-à-dire si un seul spermatozoïde pénètre l'ovule, il ne doit pas apparaître de ségrégation anormale. Une pénétration d'un trop grand nombre de spermatozoïdes pourrait peut-être aussi restreindre 1'intensité du phénomène, si la compétition se trouvait alors " diluée " et l'optimum serait probablement la pénétration simultanée d'un petit nombre de spermatozoïdes $\left.{ }^{(}{ }^{1}\right)$.

Pour tenter de vérifier le bien-fondé de notre supposition on pourrait donc essayer d'influer indirectement sur la possibilité de polyspermie, en contrôlant en particulier la dilution du sperme. Il serait aussi souhaitable de voir s'il existe, indépendamment de la dilution, des différences individuelles entre reproducteurs pour la polyspermie et d'examiner s'il s'ensuit des variations parallèles du rapport de ségrégation.

\section{Comparaison avec les résultats des autres croisements au locus $R$.}

Nous avons indiqué plus haut la différence entre nos résultats initiaux et ceux observés sur une souche de Wyandottes, où le rapport de ségrégation des types de crête est perturbé encore de façon différente suivant le sexe, mais en sens contraire de la première population étudiée.

De même, les résultats du croisement $\delta^{*}{ }^{*} R r \times q q R r$, obtenus sur diverses populations, présentent un rapport de ségrégation inversé d'un sexe à l'autre, mais différent du croisement $\delta^{*} \sigma^{*} R r \times q q r r$ (MÉRAT, I963c). Il est possible que, par exemple, des gènes, liés ou non à $R$, soient responsables de ces différences; dans l'hypothèse d'un gène lié, une inversion du linkage d'une population à une autre conduirait à une inversion du rapport de ségrégation. Il est de fait que, comme

(1) De ce point de vue, notre hypothèse peut suggérer également une interprétation des fluctuations du rapport de ségrégation d'une semaine à l'autre, la concentration du sperme, donc peut-être les conditions ou possibilités de polyspermie, pouvant varier considérablement d'un éjaculat à l'autre. 
nous l'avons indiqué (MÉRAT, I963c), une famille particulière, dans le croisement ð $R r \times \& R r$, a donné chez les mâles une proportion très nettement en sens contraire de la tendance générale; des résultats ultérieurs sur 6 populations (données non publiées) suggèrent que cette tendance peut être plus ou moins prononcée suivant la souche.

Il est possible aussi que le génotype de la mère, ou encore celui du globule polaire, au locus $R$ ou à un locus voisin, modifie le comportement des spermatozoïdes $R$ et $r$ vis-à-vis des divers types d'ovules.

\section{CONCLUSION}

Le phénomène observé ici est-il exceptionnel chez les volailles? D'autres cas de ségrégation anormale, relatifs au locus C (MÉRAT, I966c), au locus $W$ (MÉrAT, I966d) et au gène $P$ (crête en pois/simple : MÉRAT, données non publiées), suggèrent que non. D'autre part, des ségrégations aberrantes, avec perturbation de la proportion des sexes, ont été signalées chez le Dindon par Buss et Davis (r963) et chez la Poule par BrILES (I963). Aucune interprétation n'est proposée par les auteurs : on peut se demander s'il n'y a pas une analogie avec nos propres résultats.

Il est intéressant de remarquer un certain parallèle entre nos données et celles de Pronka (I965) sur le lin : là également on observe des disjonctions anormales dans un seul sexe dues non à des anomalies de la méiose, mais à une réaction dans les styles vis-à-vis des tubes polliniques.

Rę̧u pour publication en octobre 1969.

\section{REMERCIEMENTS}

Nous remercions le Dr MerRitr, Animal Research Institute, Ottawa, Canada et le Dr LAUvergNe, Station centrale de Génétique animale, C.N.R.Z., 78-Jouy-en-Josas, France, des utiles suggestions et remarques qu'ils ont bien voulu nous apporter en relisant cet article.

\section{SUMMARY}

FURTHER DATA ON SEgREgation ABNORMALITIES AT THE $R$ LOCUS OF THE HEN WHEN THE SIRE IS HETEROZYGOUS

Further results are given for an abnormal segregation ratio at the locus $R$ (rose/single comb) in the cross $\delta R r \times \& r r$. They confirm a fact already known-that there is an excess of single-comb males and a shortage of rose-comb ones as compared to the expected proportion. An analogous phenomenon may be present in data from another author (WARREN, 1948) in a linkage study. On the other hand, another population we studied shows a diverging proportion between the two sexes, which is different from that obtained in the first strain.

The data on this first population suggest time fluctuations of the segregation ratio in those families where this ratio, on the whole, deviates significantly from expectation.

Finally, an examination of simultaneous segregations at the $C$ locus (presence/absence of feather pigment) confirms that the excess of single comb males is then limited to colored zygotes $(C C$ or $C c)$.

These observations seem to confirm our previous interpretation by a mechanism of " selective fertilization ,". Moreover the results of a double segregation at loci $R$ and $C$ suggest the possibility of a direct " interaction " or " competition" between $R C$ and $r C$ sperm cells when the 
ovum has the $\mathrm{X}$ chromosome. The working hypothesis suggested is that this " competition " might occur when there is polyspermic penetration of the sperm nuclei of these two genotypes into the egg cytoplasm.

\section{RÉFÉRENCES BIBLIOGRAPHIQUES}

ARNold C. G., 1958. Selektive Befruchtung. Ergebn. Biol., 20, 67-96.

Bateman N., r96o. Selective fertilization at the T-locus of the mouse.

BRILES W. E., r963. Current status of blood groups in domestic birds. 37r-39r.

Buss E. G. and Davis G. T., 1963. Abnormal sex ratios in crossbred turkeys. Genetics, 48, 884 (Abstr.).

MÉRAT, P. 1962. Ségrégations anormales pour les allèles "crête simple " et "crête en rose " chez la poule. I-croisement ơ $\mathrm{Rr} \times$ o $\mathrm{rr}$. Ann. Biol. anim. Biochim. Biophys., 2, rog-r17.

Mérat P., r963a. Abnormal segregations for the alleles " single comb" and " rose comb" in the domestic fowl. XIe Congr. Genetics, 1, 259 (Abstr.).

MÉrat P., r $963 b$. Ségrégations anormales pour les allèles "crête simple " et crête " en rose " chez la poule. IV. Discussion d'ensemble. Ann. Biol. anim. Biochim. Biophys., 3, 133-x41.

MÉrat, P. I963c. Ségrégations anormales pour les alleles "crête simple " et "crête en rose " chez la poule. III. Croisement Rr $\times$ Rr. Ann. Biol. anim. Biochim. Biophys., 8, 125-I3I.

Mérat P., 1966a. Contribution à l'étude de la "valeur sélective " associée à quelques gènes chez la poule domestique. Thèse, Fac. Sciences, Paris.

MÉRAT P., r $966 b$. Interaction entre deux loci pour une ségrégation anormale chez la poule. Ann. Biol. anim. Biochim. Biophys., 6, 427-429.

Mérat, P. I966c. Ségrégation anormale au locus $C$ chez la poule domestique. Ann. Biol. anim. Biochim. Biophys., 6, 423-425.

Mérat P., r966d. Irrégularités des proportions mendéliennes au locus $W / w$ chez la poule domestique. Ann. Biol. anim. Biochim. Biophys., 6, 249-254.

Prko L., I96r. La polyspermie chez les animaux. Ann. Biol. anim. Biochim. Biophys., 1, 324-384.

Plonka P., 1965. L,es disjonctions anormales chez le lin cultivé, exemples de sélection gamétophytique. Ann. Génét., 8, II 2 (abstr.).

TYLER A., 196r. Approaches to the control of fertility based on immunological phenomena. J. Reproduct. Fertil., 2, 473-506.

WARREN D. C., I948. Linkage relations of autosomal factors in the fowl. Genetics, 34, 333-350. 